\title{
Introducing enteral feeding induces intestinal subclinical inflammation and respective chromatin changes in preterm pigs
}

\begin{abstract}
Aim: To analyze how enteral food introduction affects intestinal gene regulation and chromatin structure in preterm pigs. Materials \& methods: Preterm pigs were fed parenteral nutrition plus/minus slowly increasing volumes of enteral nutrition. Intestinal gene-expression and chromatin structure were analyzed 5 days after birth. Results: Enteral feeding led to differential upregulation of inflammatory and pattern recognition receptor genes, including $I L 8$ (median: $5.8,95 \% \mathrm{Cl}: 3.9-7.8$ for formula; median: $2.2,95 \% \mathrm{Cl}: 1.3-3.3$ for colostrum) and TLR4 (median: $3.7,95 \% \mathrm{Cl}: 2.6-4.8$ for formula; no significant differences for colostrum) with corresponding decondensed chromatin configurations. On histology this correlated with mild mucosal lesions, particularly in formula-fed pigs. In CaCo-2 cells, histone hyperacetylation led to a marked increase in TLR4 mRNA and increased IL8 expression upon stimulation with lipopolysaccharide (median: 7.0; interquartile range: 5.63-8.85) compared with naive cells (median: 4.2 ; interquartile range: $2.45-6.33 ; p=0.03$ ). Conclusion: Enteral feeding, particular with formula, induces subclinical inflammation in the premature intestine and more open chromatin structure in key inflammatory genes. This may increase the susceptibility for necrotizing enterocolitis.
\end{abstract}

Keywords: chromatin $\bullet$ DNA methylation $\bullet$ enteral nutrition • necrotizing enterocolitis - premature infants

In modern neonatology, necrotizing enterocolitis (NEC) is one of the most devastating acute disorders in premature infants [1] and thus became a high priority research topic over the last decades. Given that to date, no NEC-specific diagnostic biomarkers are available [2-4], the diagnosis continues to be based primarily on clinical parameters. Consequently, afflicted patients with NEC stage II or III referred for surgical evaluation require intestinal resection in up to $80 \%$ [5] with high subsequent mortality and morbidity, such as short bowel syndrome. Besides the significant impairment in quality of life, this also leads to a heavy long-term social and financial burden for society [6]. It has long been known that NEC develops in the presence of bacteria and enteral feeding and that prematurity is the most important risk factor $[7,8]$. However, there is increasing evidence suggest- ing that an altered and possibly exaggerated immature intestinal immune response of the host might be the primary factor contributing the exact sequence of events is unclear, the innate immune pattern recognition receptor Toll-like receptor 4 (TLR4) seems to play a key role, which is supported by the fact that $\mathrm{C} 3 \mathrm{H} / \mathrm{HeJ}$ mice carrying dysfunctional TLR 4 are protected against NEC [12]. Accordingly, upon activation of TLR 4 by bacterial-derived products induced inflammatory gene-expression in intestinal epithelial cells [13] as well as reduced enterocyte proliferation and impaired vascular response to hypoxia in mouse models both in vitro and in vivo have been reported [14,15]. Nevertheless, it still remains unclear why TLR4 has such a detrimental effect and whether it is per se upregulated in the immature gut or whether additional trigto the development of NEC [9-11]. Although
Rhea Willems', Lukasz Krych ${ }^{2}$ Verena Rybicki', Pingping Jiang ${ }^{3}$, Per T Sangild ${ }^{3}$, René L Shen ${ }^{3}$, Kai O Hensel', Stefan Wirth', Jan Postberg' \& Andreas $C$ Jenke*,1 'Department of Paediatrics, HELIOS Medical Centre Wuppertal, Centre for Clinical \& Translational Research (CCTR), Faculty of Health, Centre for Biomedical Education \& Research (ZBAF), Witten/Herdecke University, Heusnerstr. 40,42283 Wuppertal, Germany 2Department of Food Science, Faculty of Science, University of Copenhagen, Frederiksberg, Denmark

${ }^{3}$ Comparative Pediatrics \& Nutrition, Department Clinical Veterinary \& Animal Science, Copenhagen University Hospital (Rigshospitalet), University of Copenhagen, Denmark

*Author for correspondence: Tel.: +49020 28963825 Fax: +4902028963804 andreas.jenke@uni-wh.de 
gers are necessary for its activation. Thus a genomewide expression analysis in premature human infants with NEC revealed no differential expression of TLR4, relative to premature infants with focal intestinal perforation [16]. Also, no mutations in TLR4 have been associated with NEC to date; and in pigs, TLR4 mRNA was not upregulated in preterm versus term pigs [17]. A possible explanation may be that TLR4 expression is modulated by environmental factors, such as feeding and bacterial colonization. Theoretically, these might induce changes in chromatin structure and $\mathrm{CpG}$ signaling, possibly destabilizing the intestinal homeostasis and thus creating the basis for an inappropriate immune response upon further exposure to bacterial antigens. Postnatal, introduction of enteral nutrition and bacterial colonization represent the most important environmental changes for the GI tract also playing a key role in NEC development both in humans [18] and pigs [19]. Importantly, early but slow introduction of enteral feeding using mother's milk or colostrum is associated with significantly less NEC, relative to formula.

Against this background, we aimed to provide new insights into how introduction of enteral nutrition influences the transcriptional regulation in the intestine and how this contributes to the development of NEC. We analyzed the in-depth intestinal responses to a gentle enteral nutrition (EN) regimen as currently used in many European neonatal intensive care units with early and slow introduction of enteral feeds in association with supportive parenteral feeding. Therefore, we used preterm pigs since they tend to develop NEC spontaneously if fed larger amounts of formula particularly if feeding is advanced rapidly after preterm birth, closely reflecting human disease [6,19-20]. This unique approach enabled us to analyze digestion- and inflammationrelated genes, together with their DNA methylation and chromatin structure in the intestinal epithelium prior to development of clinical symptoms of NEC.

\section{Materials \& methods}

Porcine model, enteral feeding \& tissue collection

Fifteen preterm pigs (landrace $\times$ large white $\times$ duroc) were delivered by caesarean section on day $105(90 \%)$ of gestation from one sow as previously described [19] and stratified according to birth weight and sex. Due to species-dependent organ maturation, the intestine matures relatively late in pigs and a preterm pig intestine at $90 \%$ gestation therefore represent the preterm human infant intestine at approximately $70 \%$ gestation $[19,21]$. Housing in incubators with regulated temperature and oxygen supply, umbilical and orogastric catheterization and passive immunization were carried out as previously described [22].
The pigs were randomly assigned to either receive total parenteral nutrition (TPN) via the catheter fitted in the umbilical artery $(n=5)$ or minimal enteral nutrition with bovine colostrum $(n=5)$ or preterm infant formula $(n=5)$. Bovine colostrum was used since this diet has been shown to elicit similar responses as porcine colostrum in the premature intestine of pigs [22] and is currently tested for use in human premature infants during the first days after birth [23]. All animals were also provided with supplementary parenteral nutrition (PN) to match the energy intake of the TPN group. The PN solution (Kabiven, Fresenius Kabi, Bad Homburg, Germany) for all groups was prepared and administered as previously described and modified to meet the nutrient requirements of preterm pigs [20]. TPN pigs received $\mathrm{PN}$ at a rate of 3, 4, 5 and $6 \mathrm{ml} / \mathrm{kg}$ bodyweight/h on days $1,2,3$ and 4 , respectively. The enteral feeding volume was $2,4,6,8 \mathrm{ml} / \mathrm{kg}$ bodyweight $/ 3 \mathrm{~h}$ on days $1-4$. Formula adopted was a $16.5 \%$ solution of enfalac premature powder (Mead Johnson Nutrition, IL, USA) in bottled water and the colostrum feeding was a $17 \%$ solution of bovine colostrum powder (Biofiber-Damino, Gesten, Denmark). All procedures were approved by the National Council on Animal Experimentation in Denmark (protocol number 2012-15-293400193).

Pigs were euthanized and the GI tract (GIT) was sampled on the fourth day after initiation of EN. Clinical signs of NEC were recorded according to our macroscopic NEC evaluation system as described previously [19]. Three regions of small intestine, stomach and colon were graded for pathology using a macroscopic NEC lesion score (1: no or minimal focal hyperemic enterocolitis; 2 : mild focal enterocolitis; 3 : moderate locally extensive enterocolitis; 4 : severe focal enterocolitis; 5: severe locally extensive hemorrhagic and necrotic enterocolitis or 6: severe extensive hemorrhagic and necrotic enterocolitis). Pigs with a score of 3 or more in any of the intestinal regions (Prox, Mid, Dist or Colon) were classified as a case of NEC (Supplementary Data 1; see online at www.futuremedicine.com/doi/full/10.2217/EPI.15.13). One to two 1 $\mathrm{cm}$ sections of the middle small intestine were stored in $4 \%$ paraformaldehyde for later histological analysis, two sections were snap frozen in liquid nitrogen and stored at $-80^{\circ} \mathrm{C}$ for later intestinal brush border enzymatic activity assays of lactase, aminopeptidase A (ApA) and dipeptidyl peptidase 4, RT-qPCR and epigenetic analysis, respectively.

We decided to primarily focus our analyses on the mid intestine for two main reasons: The small feeding volumes used in this study were expected to have more immediate effects on the proximal and middle part of the intestine when compared with the lower 
parts; in preterm pigs the mucosal immune system including Peyer's patches are not clearly evident in the distal intestine but are well developed in the mid intestine which thus probably best represents the premature human intestine regarding structural, functional and immunological responses.

\section{Oligonucleotides}

Primers utilized in this study are listed in Supplementary Data 2.

\section{Sample pretreatment}

Porcine intestinal specimens stored in liquid nitrogen were pulverized prior to the purification of RNA, gDNA and chromatin using a stainless steel mortar and piston (Cellcrusher, Cork, Ireland) prechilled in liquid nitrogen. Only tissue samples without microscopic signs of leukocyte invasion were used for further analysis.

\section{High-throughput sequencing of the gut microbiota}

The fecal microbiota of formula and colostrum-fed pigs was determined using tag-encoded 16S rRNA gene MiSeq-based (Illumina, CA, USA) pair-ended highthroughput sequencing. DNA storage condition, cellular DNA extraction and sequencing library preparation steps were conducted as previously described [24]. Quantitative Insight Into Microbial Ecology open source package (1.7.0) and CLC Genomic Workbench 7 (CLC bio, Arhus, Denmark) were used to analyze the sequencing data as previously described [24]. The relative distribution of the genera registered was calculated for unified ( $85 \%$ of the most indigent sample) and summarized in the family and genus level OTU tables. Two samples were discarded due to the low number of reads. The alpha diversity measures expressed with an observed species (sequence similarity 97\% OTUs) value were computed for rarefied OTU tables $(28,000)$ using the alpha rarefaction workflow. Differences in alpha diversity were determined using a t-test-based approach employing the nonparametric (Monte Carlo) method (999 permutations) implemented in the compare alpha diversity workflow. The ANOVA test performed based on 999 rarefied OTU tables was used to determine quantitative (relative abundance) association of genus level OTUs with one of the two categories.

\section{Gene-expression analyses}

Prior to gene-expression analyses RNA was isolated from at least three biological replicates using Trizol reagent (Sigma-Aldrich, MO, USA) upon manufacturer's recommendations. RNA integrity was validated utilizing an Agilent Bioanalyzer 2100 with the Agilent RNA 6000 Nano Kit for microcapillary electrophore- sis. Using the RT2 First Strand kit (Qiagen/SABiosciences, Venlo, The Netherlands), cDNA templates were synthesized from $1 \mu \mathrm{g}$ RNA. Gene-expression was analyzed from these cDNAs using primer pairs as listed in Supplementary Data 2 with Quantitect SYBR Green Taq polymerase mastermix (Qiagen) on a Corbett Rotor-Gene 6000 qPCR device. PCR conditions were as follows: $95^{\circ} \mathrm{C}$ for $15 \mathrm{~min}, 40$ cycles of $\left(95^{\circ} \mathrm{C}\right.$ for $15 \mathrm{~s}$, $60^{\circ} \mathrm{C}$ for $30 \mathrm{~s}$ ). Melting of PCR product was performed using a temperature gradient from $55-95^{\circ} \mathrm{C}$, rising in $0.5^{\circ} \mathrm{C}$ increments. For relative comparative quantification of gene-expression fold changes we utilized the $\Delta \Delta \mathrm{Ct}$ method [25] using at least three housekeeping genes for normalization.

\section{DNA CpG methylation analyses}

For the assessment of DNA methylation in the promoter regions of differentially regulated genes, we combined CpG methylation-sensitive restriction endonucleolytic digests using HpaII (New England Biolabs, MA, USA) in combination with quantitative real time PCR. In addition, methylation-insensitive MspI (New England Biolabs) was used to control the integrity of the restriction enzyme recognition motif in each sample. Three sites within the H19 promoter were selected as DNA methylation-positive controls. Genomic imprinting of the H19 gene involves DNA methylation which leads to subsequent DNA hemimethylation [26]. Restriction digests were incubated at $37^{\circ} \mathrm{C}$ for $1 \mathrm{~h}$ prior to heat inactivation for $15 \mathrm{~min}$ at $95^{\circ} \mathrm{C}$, simultaneously activating hot start Taq polymerase. PCR conditions were as described above for qPCR gene-expression analysis. Finally, melting of PCR product was performed using a temperature gradient from $55-95^{\circ} \mathrm{C}$, stepwise rising in $0.5^{\circ} \mathrm{C}$ increments. For relative comparative quantification of template recovery after methylation sensitive restriction digests we applied a variation of the $\Delta \mathrm{Ct}$ method to compare the relative ratio of HpaII-digested DNA with undigested template DNA:

- $\Delta \mathrm{Ct}=\mathrm{Ct}_{\mathrm{HpaII}-\text { digested DNA }}-\mathrm{Ct}_{\text {undigested DNA; }}$

- DNA recovery [\%] $=\mathrm{AE}^{-\Delta \mathrm{Ct}} \times 100 \%$, whereby $\mathrm{AE}$ is the amplification efficiency determined for each primer pair;

- Methylated CpG [\%] = 100\% - DNA recovery [\%].

Benzonase endonuclease hypersensitivity assay

To analyze chromatin structure at specific sites of interest we applied the tissue accessible chromatin method [27] in combination with quantitative real time PCR, since Benzonase endonuclease hypersensitive sites frequently correlate with actively transcribed genes and are markers of cis-regulatory DNA elements such 
as promoters, enhancers and locus control regions [28]. Briefly, liquid nitrogen-chilled, pulverized tissue samples were treated with the DNA endonuclease benzonase (Sigma-Aldrich). Next, DNA was purified by phenol: chloroform: isoamylic alcohol (25:24:1) extraction and precipitated with ethanol. Recovery of template DNA was compared with nondigested DNA analyzed using quantitative PCR as described above.

\section{Trichostatin A \& lipopolysaccharides stimulation in $\mathrm{CaCo}-2$}

Human CaCo-2 cells were maintained in Dulbecco's Modified Eagle's Medium enriched with 10\% fetal bovine serum, $10 \%$ L-glutamine, 50 units $/ \mathrm{ml}$ penicillin and $50 \mu \mathrm{g} / \mathrm{ml}$ streptomycin in a humidified atmosphere $\left(5 \% \mathrm{CO}_{2}\right)$ at $37^{\circ} \mathrm{C}$. The medium was supplemented with $330 \mathrm{nM}$ trichostatin A (TSA) (Sigma-Aldrich) and/or $50 \mathrm{ng} / \mathrm{ml}$ lipopolysaccharides (LPS) from Salmonella enterica serotype typhimurium (Sigma-Aldrich) as follows: treatment with TSA only for $1 \mathrm{~h}$; treatment with LPS only for $2 \mathrm{~h}$ or treatment with TSA for $1 \mathrm{~h}$ followed by LPS exposure for $2 \mathrm{~h}$. RNA-isolation, integrity testing and cDNA synthesis were performed as described above. PCR conditions were as follows: $95^{\circ} \mathrm{C}$ for $15 \mathrm{~min}, 50$ cycles of $\left(95^{\circ} \mathrm{C}\right.$ for $15 \mathrm{~s}, 60^{\circ} \mathrm{C}$ for $30 \mathrm{~s}$ ). Melting point analyses were performed utilizing qPCR as described above. All utilized primers are listed in Supplementary Data 2. The mitochondrial gene ATP 6 was used for normalization as it has no chromatin organization.

\section{Statistical analysis}

mRNA quantification levels of all analyses were obtained from triplicate real-time PCR measurements. Data are presented as medians \pm interquartile ranges (IQR), minima and maxima. Testing for significant differences between groups was performed using the one-way ANOVA test for values with Gaussian distribution and the Mann-Whitney test for values without Gaussian distribution. When significant differences were found, the post hoc Bonferroni correction was applied to correct for use of multiple comparisons. The Kolmogorov-Smirnov test was utilized to rule out non-Gaussian distribution. In addition, one-way ANOVA testing was always combined with Bartlett's of equal variance. Values of $p<0.05$ were considered statistically significant. All analyses were performed with GraphPad version 5.01 (CA, USA).

\section{Results}

Enteral nutrition induces subclinical, diet-dependent intestinal epithelial inflammation

When animals were sacrificed on day 5 , none of them had any clinical symptoms of NEC (vomiting, abdomi- nal distention, apnea, discoloration, bloody stools). Four out of the five formula-fed pigs but none of the colostrum-fed pigs showed clear NEC-like lesions in the colon region (NEC score 4 or 5) and were thus diagnosed with NEC despite the apparent absence of clinical symptoms prior to euthanasia. Characteristics of the pigs are given in Supplementary Data 1. For all pigs, histological examination of the mid small intestine revealed an intact intestinal epithelium with only mild mucosal inflammation and without any obvious NEClike lesions in any of the three groups, although pigs fed with enteral diets had the most dense mucosal epithelium and longest villi (Figure 1A). This was also reflected by a significantly higher intestinal weight (Figure 2A), and intestinal weight per length $(84 \mathrm{vs} 68 \mathrm{mg} / \mathrm{cm}$, $\mathrm{p}<0.05)$ in both groups fed supplemental EN, relative to TPN. Lactase activity was markedly reduced in the pigs supplemented with formula (Figure 2B), while both groups receiving enteral nutrition showed elevated activity of dipeptidyl peptidase 4 (Figure $2 \mathrm{C}, \mathrm{p}=0.08$ ) and ApA (Figure 2D), relative to TPN.

We also analyzed the composition of the microbial flora in the colon luminal content comparing formula with colostrum fed pigs. The sequences purged from chimeric reads yielded 673,377 giving an average of 84,172 sequences per sample $(\mathrm{min}=33,171$; $\max =125,526 ; S D=29,416$ with a mean sequence length of $439 \mathrm{bp}(\mathrm{SD}=12 \mathrm{bp})$. Alpha diversity measures expressed with observed species index showed no differences between the two categories (data not shown). The majority (>99\%) of all reads were distributed between phyla Firmicutes and Proteobacteria in both categories accounting respectively for 43 and $57 \%$ in the formula-fed group, and 7 and $92 \%$ in the colostrum-fed group. These differences however did not reach significance. The genus level analysis indicated differences between the two categories reflected in the relative abundance of genus Klebsiella (proteobacteria), Enterococcus (firmicutes) and unclassified genus from Enterobacteriaceae family (proteobacteria). However, only the latter difference has reached significance (ANOVA $\mathrm{p}<0.001^{* * *}$ ) (Figure 3 ).

Nutrition induces diet-specific changes in geneexpression \& chromatin signatures of genes related to inflammation \& innate immunity The expression profiles of several genes differed significantly between TPN and supplemental enteral nutrition in general and between formula and colostrum feeding in particular as determined by analyses of relative mRNA enrichment by quantitative real time PCR (Figure 4). Relative mRNA copy numbers of inflammatory and pattern recognition receptor (PRR) genes, such as IL8, TLR2, TLR4, REG3A, an antimicrobial 


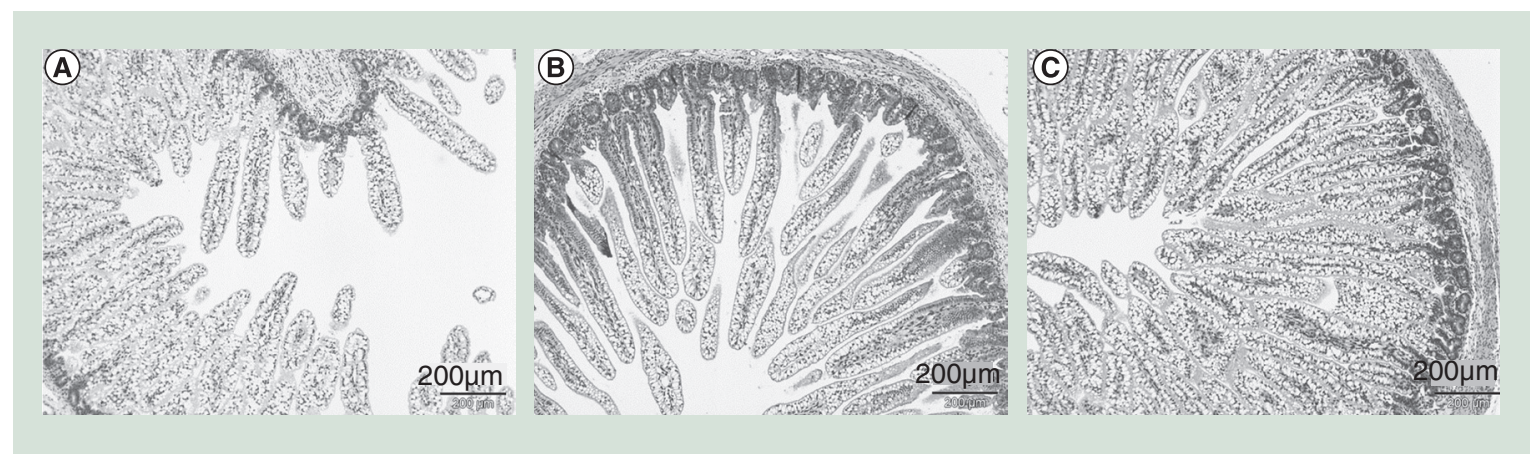

Figure 1. Representative histological pictures of the intestinal epithelium in preterm 5-day-old pigs. (A) Preterm pigs were fed exclusively with total parenteral nutrition, (B) parenteral nutrition plus formula or (C) parenteral nutrition plus colostrum. The micrographs of the small intestine show no necrotizing enterocolitis-like lesions although the mucosal mass and density was higher in both groups where parenteral nutrition was supplemented with enteral nutrition (formula, colostrum), relative to total parenteral nutrition alone.

protein in control of bacterial proliferation [29] and $L Y Z$, an enzyme catalyzing hydrolysis of bacterial cell wall components, were elevated in both groups, compared with TPN (Figure 4A \& B). This effect was significantly more pronounced for most inflammatory and innate immunity genes in the formula versus colostrum pigs (Figure $4 C$ ), with the only exception being that of TLR2, which was less expressed in formula-fed pigs. Interestingly, mRNA encoding the anti-inflammatory cytokine IL10, which is known to block NF- $\mathrm{KB}$ activity and be involved in the regulation of the JAK-STAT signaling pathway, was significantly enriched in pigs fed colostrum and downregulated in formula-fed piglets when compared with pigs fed PN alone (Figure 4). To answer the question whether the observed changes in gene-expression were also associated with changes in the chromatin structure for specific genes of interest, we analyzed endonuclease hypersensitivity sites. Importantly, almost all inflammatory and innate immunity genes, previously found to be differentially upregulated in formula-fed pigs, colocalized with chromatin segments exhibiting significantly higher benzonase endonulcease hypersensitivity, particularly TLR4 and IL8. The only exception was TLR2 (Figure 5A). Moreover, the observed differences in benzonase endonulcease hypersensitivity also seemed to correlate well with similarly directed changes in DNA methylation profiles (Figure 5B). Again no differences were found at the TLR2 locus (Figure 5B) or at any of the sites examined for other genes of interest (data not shown).

\section{Chromatin decompaction through impaired histone deacetylation increases TLR4 expression in intestinal epithelial cells \& leads to elevated IL8 mRNA accumulation upon subsequent LPS stimulation.}

Next, we analyzed whether an induced open chromatin conformation in intestinal epithelial cells, specifi- cally at the TLR4 locus might influence the inflammatory response upon stimulation with LPS. This was done using $\mathrm{CaCo}-2$ cells, since under specific conditions $\mathrm{CaCo}-2$ cells functionally resemble enterocytes in the small intestine [30] and currently represent one of the best characterized in vitro models of the intestinal barrier [31]. CaCo-2 cells were treated with the histone deacetylase (HDAC) TSA and their inflammatory response was compared with naive $\mathrm{CaCo}-2$ cells. Treatment with TSA resulted in significantly higher mRNA copy numbers for TLR4, whereas it had no detectable effect on TLR2 and IL8 mRNA (Figure 6). Stimulation with LPS led to a significant increase in IL8 mRNA copy numbers in both TSA pretreated and naïve $\mathrm{CaCo}-2$ cells, but the observed increase was significantly higher in TSA pretreated cells (median: 7.0; IQR: $5.63-8.85$ vs 4.2; IQR: $2.45-6.33 ; \mathrm{p}=0.03$ ) (Figure 6). No differences were noted for IL6 mRNA copy numbers, which were found to be decreased upon TSA treatment and/or upon LPS stimulation, when compared with naïve $\mathrm{CaCo}-2$ cells.

\section{Discussion}

The protective effect of breast milk toward the development of NEC was first reported several decades ago [32], but nevertheless it has remained unclear how the timing (early vs late), amount (slow vs rapid) and diet (breast milk vs formula) of the first enteral feeds for preterm neonates affect NEC sensitivity [33]. In this study, we used a preterm pig model to investigate the effect of an early and slow introduction of enteral feeds on the intestine as used in many neonatal intensive care units for preterm infants. This slow feeding advancement generally preserved small intestinal structure and functions and induced only mild NEC lesions (in the colon, and only in formula-fed pigs) in contrast to more rapid advancement which has been shown to induce clinical NEC symptoms and mac- 
(A)

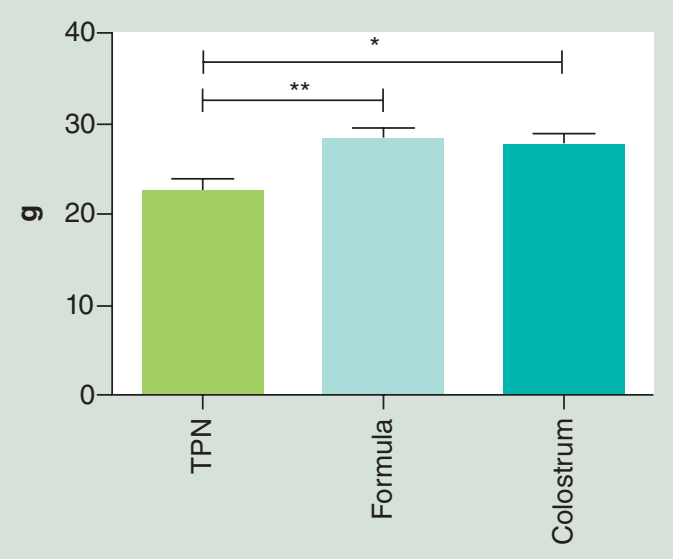

(C)

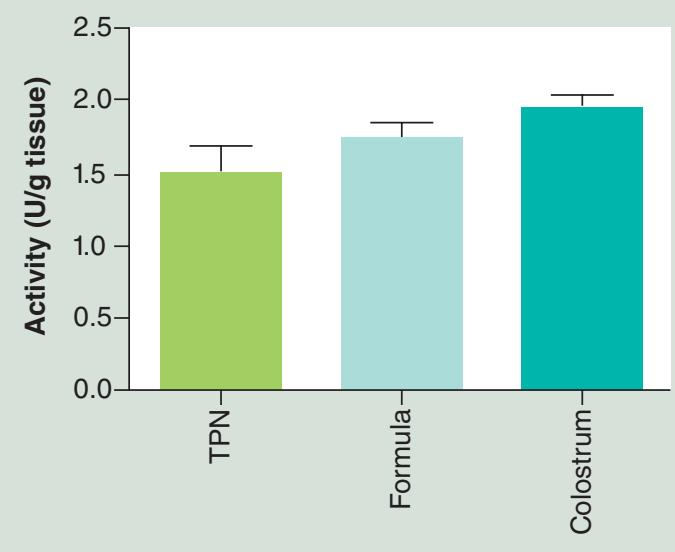

(B)

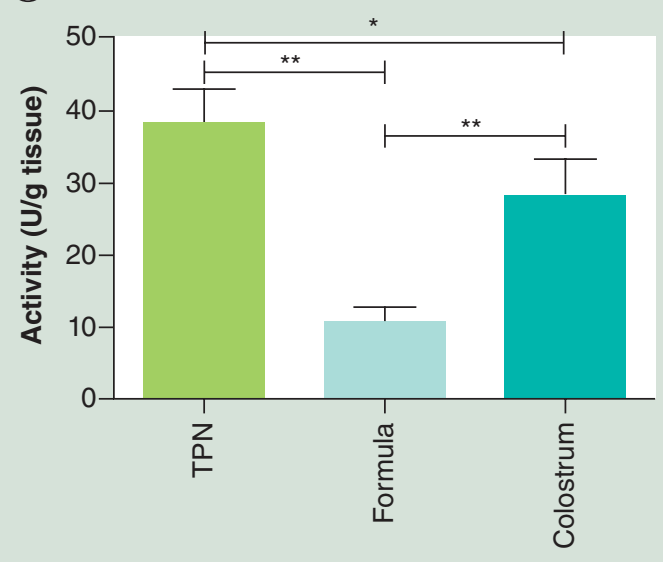

(D)

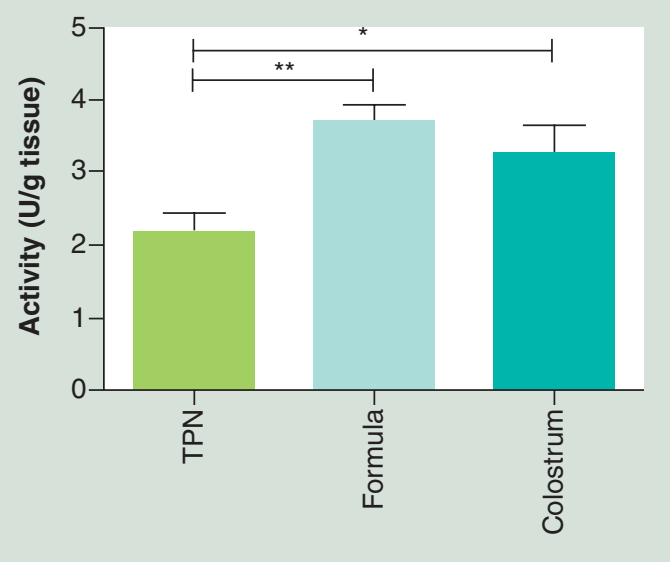

Figure 2. Influence of diet on intestinal weight and digestive enzyme activities in preterm 5-day-old pigs.

Preterm pigs were fed exclusively with TPN, parenteral nutrition plus formula (formula) or parenteral nutrition plus colostrum (colostrum). (A) Small intestinal weight and (B) enzymatic activities of lactase, (C) dipeptidyl peptidase 4 and (D) ApA differed significantly among the three groups (means \pm standard error of mean). * $\mathrm{p}<0.05 ; * * \mathrm{p}<0.01$.

TPN: Total parenteral nutrition.

roscopic intestinal pathology in preterm pigs, especially using formula diets [34]. Thus, we were able to characterize the intestinal epithelial response on the gene-expression and chromatin level to the first enteral feeds prior development of any clinical signs of NEC. We also compared an infant formula with a bovine colostrum product that has previously been shown to protect against NEC in newborn preterm pigs. The results enabled us to provide representative data on how the first small amounts of enteral feeds can shape the epigenetic landscape and consequently influence the transcription of genes in the premature intestinal epithelium and furthermore, how these changes might influence the susceptibility for NEC development.

Similar to previous studies in preterm pigs, using rapid transition to higher amounts of enteral formula or colostrum (120 ml/kg/day) [20], we observed lowered intestinal mass and activity of brush border enzymes such as dipeptidyl peptidase 4 and ApA in TPN-fed pigs when compared with the enteral feeding groups. This most likely reflects lack of enteral stimulation of tissue metabolism and blood flow. Interestingly, lactase activity was specifically reduced in pigs fed small amounts of enteral formula, even in the absence of any NEC lesions in the small intestine. Probably, intestinal lactase activity was particularly sensitive to the suboptimal formula diet because this enzyme - in contrast to other brush border enzymes - is most abundantly expressed in the proximal and middle intestine by the oldest enterocytes on the villus tips that may first show the effects of an adverse diet response. This reduced lactase activity may facilitate bacterial overgrowth due to an increased intraluminal availability of undigested carbohydrates thus predisposing to later development of NEC [20,35].

More importantly, enteral feeds, and formula feeding in particular, induced subclinical inflammation in 
the intestinal epithelium with significant upregulation of inflammatory genes, such as IL8 and PRR, such as TLR4. This translated to corresponding changes in epigenetic signatures. Thus, DNA of upregulated genes, such as IL8 and TLR4, was significantly more sensitive to benzonase, which preferentially digests DNA in regions of low nucleosome occupancy. Since such endonuclease hypersensitive regions directly reflect the accessibility of the genome [28], IL8 and TLR4, among others, seemed to be localized in decondensed, putatively activated chromatin regions, which might be enhanced depending on the type of enteral nutrition during the first days after preterm birth. Moreover, lower levels of $\mathrm{CpG}$ methylation were particularly observed for IL8 after formula feeding, providing additional indication for long lasting gene activation, since differential $\mathrm{CpG}$ signaling is frequently associated with long-term changes in the regulation of gene-expression [36]. Such a baseline activation of inflammatory and PRR genes fits well with the current concept of an exaggerated innate immune response as key factor for NEC [12,14-15,37-38], particularly since the observed nutritional effect also involves TLR4. Since to date, no genetic markers could be associated with NEC, this diet-dependent activation could provide at least a partial explanation for the exaggerated innate immune response observed in the mouse model [37]. These results also help to understand why TPN-fed preterm neonates are less sensitive to NEC, although continued TPN has many negative consequences for the immature intestine, including decreased mucosal growth and defense. In fact, in this study, TPN led to a decreased expression of antimicrobial peptides $R E G 3 A$ and $L Y Z$. This most likely reflects Paneth cell dysfunction thus increasing the susceptibility to mucosal bacterial invasion, as reported for the mature intestine [39]. Possibly such Paneth cell dysfunction plays an important role in the pathogenesis of NEC as recently suggested by McElroy et al. [40]. In consequence, optimization of postnatal nutrition in the premature intestine is obviously more complicated, particularly since it still remains unclear whether early trophic feeding in human premature infants is beneficial or not [41].

Preterm pigs fed colostrum showed a less pronounced intestinal expression of inflammatory and PRR genes and a higher expression of the anti-inflammatory cytokine IL10, relative to pigs-fed formula or parenteral nutrition alone. Remarkably, IL1O can block NF- $\kappa \mathrm{B}$ activity as an important regulator of immunity and inflammation, which can also be activated by TLR4 stimulation [42]. This finding of increased IL1O expression upon colostrum feeding might thus explain the previously reported reduced NF- $\kappa \mathrm{B}$ activity in the presence of upregulated TLR4 in colostrum-fed pigs [43]. At the same time, it partly resolves the contradictory results between the mouse model showing a TLR4-mediated exaggerated innate immune response at the center of NEC pathogenesis [37] and the porcine data demonstrating higher TLR4 expression in NECprotected colostrum-fed pigs $[17,43]$. Finally, it provides a reasonable explanation for the protective effect of colostrum in the premature pig model [19] and possibly for the protective effect of breast milk in humans [44]. At this point it is important to mention that we also observed some variations in the composition of colonic microbiota between colostrum and formula-fed pigs. Even though statistically not significant except for one genus, these variations might have partially contributed to the observed differences in gene-expression and chromatin conformation between colostrum and formulafed pigs. However, this finding was surprising since in a similar study feeding with higher volumes of enteral feeds only showed marginal differences in bacterial colonization between formula- and colostrum-fed preterm

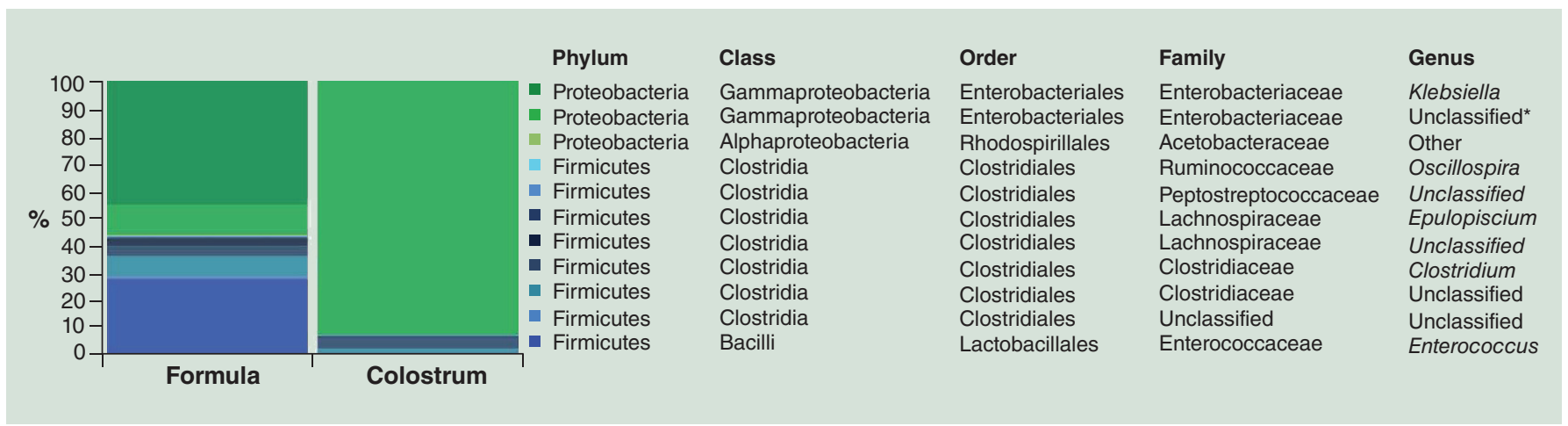

Figure 3. The average relative distribution of the major gut microbiota genera $(>0.1 \%$ in both categories) determined for formula $(n=4)$ and colostrum-fed $(n=4)$ pigs. Taxa denoted as 'unclassified' means that the reference database does not have an official taxonomy for this cluster. Taxa denoted as 'other' (unidentified), indicates ambiguity in the assignment meaning that more than one bacterial taxa could be assigned to this cluster. An unclassified genus within the Enterobacteriaceae family was the only taxon significantly different between the two categories (ANOVA $p<0.001 *$ ). 
Research Article Willems, Krych, Rybicki et al.

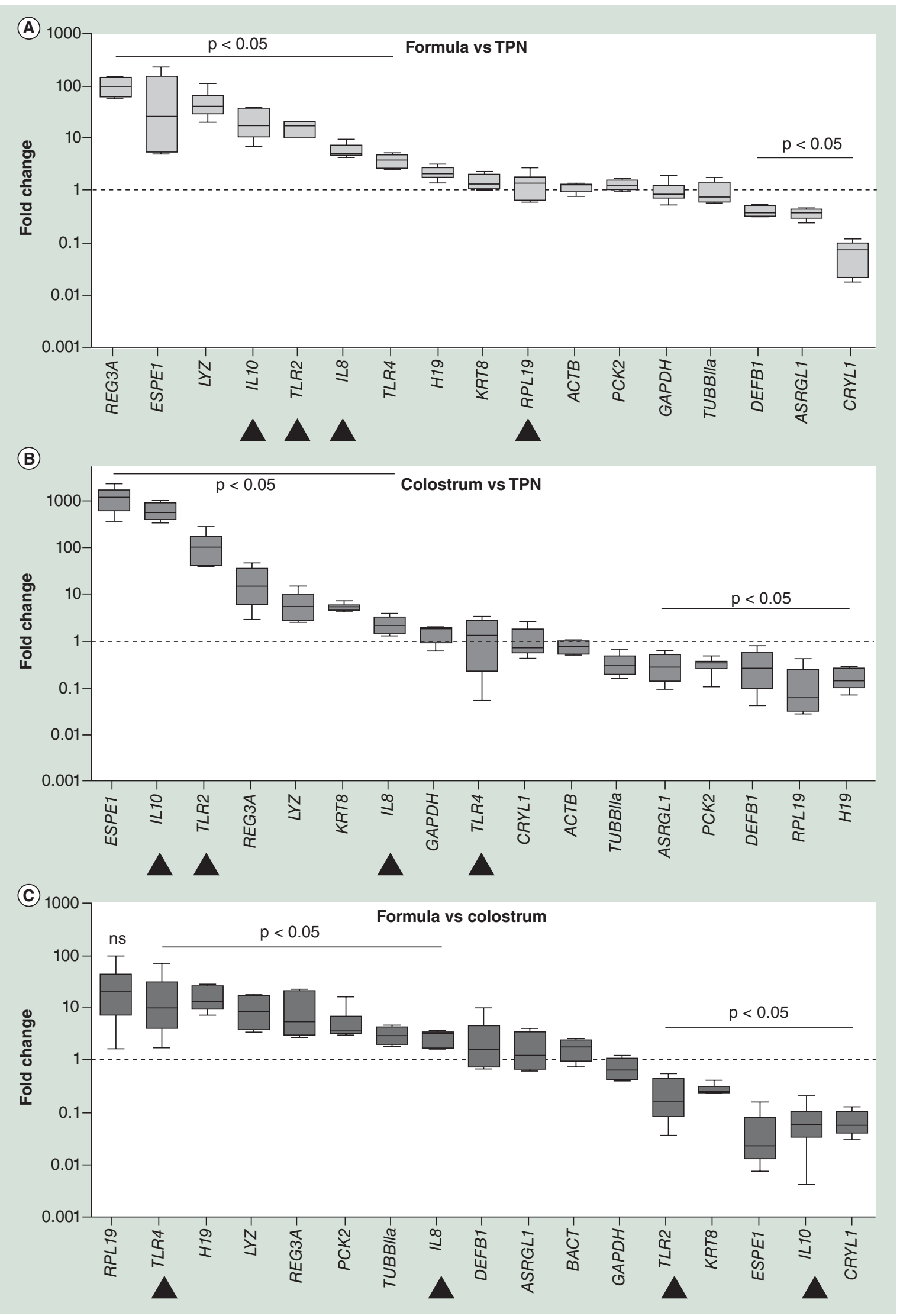


Figure 4. Influence of diet on gene-expression profiles in the intestinal epithelium of preterm 5-day-old pigs (see facing page). Preterm pigs were fed exclusively with parenteral nutrition (TPN), parenteral nutrition plus formula (Formula) or parenteral nutrition plus colostrum (colostrum). Levels of mRNA normalized to GAPDH and ACTB were compared among treatments. Inflammatory and innate immunity genes were substantially upregulated in both (A) formula and (B) colostrum fed pigs when compared to TPN. This effect was much stronger in the formula fed when compared to the (C) colostrum fed pigs. Data are presented as medians \pm interquartile ranges, minima and maxima. All experiments were performed in technical triplicates. Arrow heads indicate the most relevant changes.

TPN: Total parenteral nutrition.

pigs [45]. Moreover, bacterial colonization of the gastrointestinal mucosal surfaces in mammals begins at birth and does not initially appear to depend much on diet but rather on host-response mechanisms and the maternal microbiota in the first week of life $[46,47]$. Only later in life the relatively stable gut microbial composition may be more influenced by the type of enteral nutrition [48]. Thus the relevance of the observed variations in colonic microbiota in this study - particularly considering the low number of animals - currently remains unclear but warrants further evaluation.

In the last part of this study, we aimed to assess the possible influence of an open chromatin conformation on the TLR4-mediated innate immune response of the intestinal epithelium. We could show that inhibition of HDAC using TSA, inducing chromatin hyperacetylation and an open chromatin structure [49], led to increased TLR4 expression in $\mathrm{CaCo}-2$ cells, whereas $T L R 2$ expression remained unaffected. This is in accordance with our observations from the animal model. Strikingly, subsequent stimulation with LPS, the major component of Gram-negative bacteria that activates TLR4 [50] elicited a significantly higher inflammatory response as measured by relative IL8 mRNA copy numbers in TSA pretreated $\mathrm{CaCo}-2$ cells. In contrast, ILG expression remained almost unchanged upon stimulation with LPS which is in line with a previous report using the same cell line [51]. We also found a significant decrease in IL6 mRNA expression upon TSA treatment, confirming that TSA treatment has no effect on IL6 promoter accessibility as previously reported [52]. Even though speculative, the observed decreased ILG mRNA levels might thus result from an upregulation of counteracting cytokines, such as IL10, known to be upregulated in response to HDAC inhibition [53].

\section{Limitations of this study}

The most important limitation of this study is the lack of data from preterm infants. Even though preterm pigs and infants share many physiological and intestinal similarities, the immature porcine gut and immune system (innate as well as adaptive) still differs from those in preterm infants. Consequently, larger studies, preferably using infant intestinal samples, are necessary to confirm the findings from this study. Also, to prove that the observed intestinal responses to enteral nutrition eventually lead to NEC, it is necessary to replicate the findings in a larger group of animals followed for a longer time with serial intestinal samples for each individual. Finally, we only analyzed the microbiota from the colon luminal content and not from the mid intestine. Even though it is reasonable to assume that differences in mid intestine microbiota translate to colonic microbiota, the exact role of the microbiota for the observed effects need further study. Nevertheless, we are convinced that optimization of the enteral nutrition regimen in the days and weeks just after preterm birth is a key tool to reduce the high susceptibility to NEC.

\section{Conclusion}

In summary, here we provide evidence that enteral nutrition might at least partially be the basis for the observed exaggerated innate immune response seen in NEC. We demonstrated that even a gentle enteral feeding regimen with a gradual increase in enteral feeds as used by many neonatal intensive care units induces subclinical inflammation in the premature intestinal epithelium with subsequent changes in chromatin conformation in inflammatory and PRR genes. Importantly, these changes occurred in the absence of any clinical symptoms suggestive of NEC which rarely develop until preterm pigs or infants approach full enteral feeding (e.g., $120-180 \mathrm{ml} / \mathrm{kg} /$ day). Also, this inflammatory response was much more pronounced using infant formula, as first diet, compared with bovine colostrum.

\section{Future perspective}

We have recently initiated preliminary studies on preterm infants using bovine colostrum as the first enteral diet, prior to the later transition to full feeding with formula or breast milk. Together with additional studies these will help to further characterize the influence of enteral nutrition in modulating intestinal gene-expression and chromatin structure. These data will then allow us to adapt enteral nutrition for premature infants possibly even in a personalized way thus reducing the risk for intestinal dysfunction and NEC in particular. For this process of personalized medicine a detailed genetic characterization of NEC susceptibility would be highly desirable. 


\section{Author contributions}

R Willems, V Rybicki, P Jiang, RL Shen and J Postberg have performed the experiments and analyzed the data. A Jen- ke wrote the manuscript. P Jiang, RL Shen and P Sangild were responsible for the animal facility and collected the tissue specimens. P Sangild, S Wirth and K Hensel critically

(A)

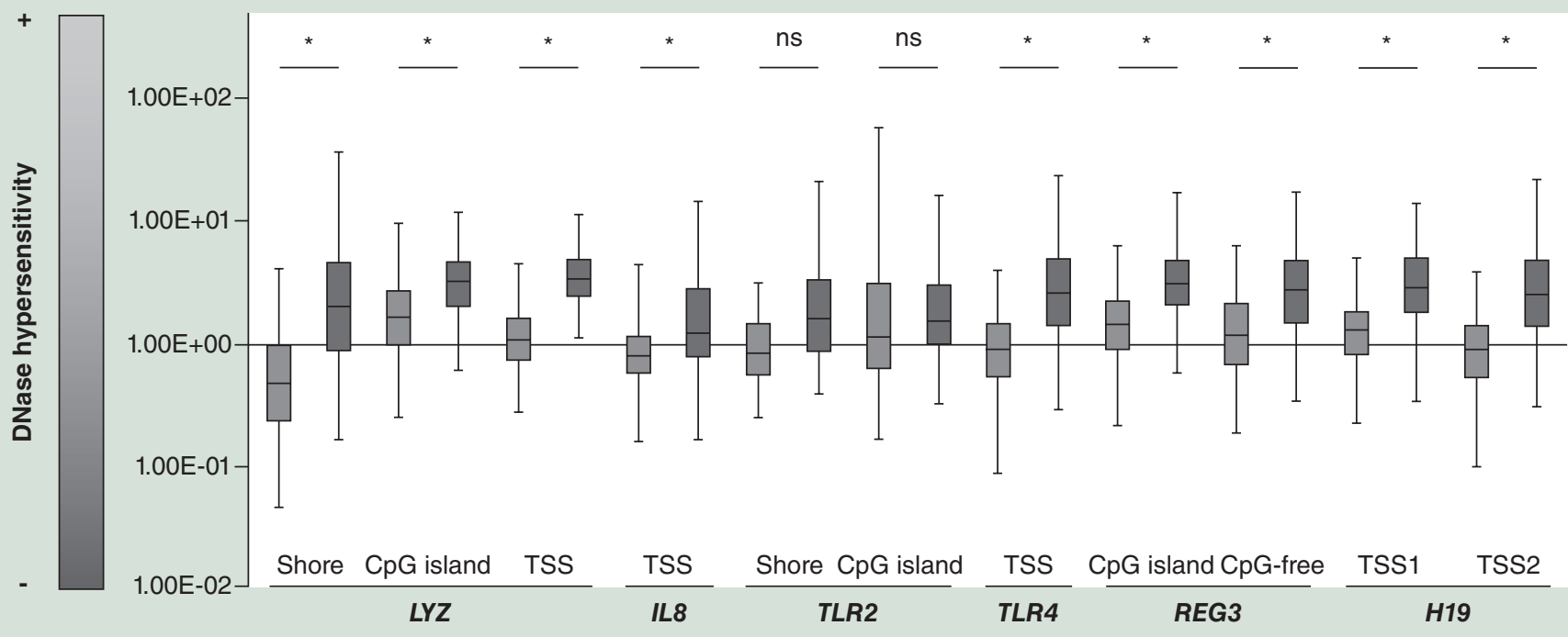

Colostrum $\square$ Formula

(B)

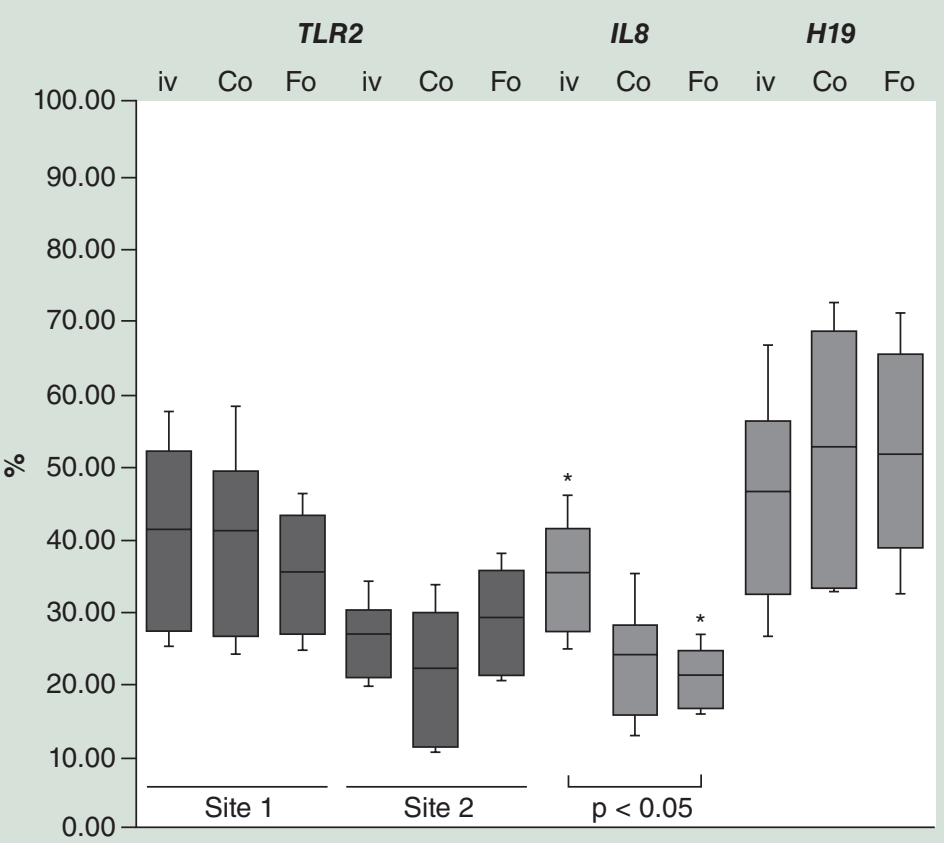

Figure 5. Influence of diet on intestinal chromatin configuration and CpG methylation. Genes upregulated in formula (dark grey), compared with colostrum (light grey) piglets, such as IL8, TLR4, REG3A and LYZ, were associated with a significantly more open chromatin conformation. (A) TPN-fed piglets were used as reference the groups supplemented with either formula or bovine colostrum. (B) CpG methylation patterns also correlated with $m R N A$ levels. The IL8 locus was significantly less methylated in pigs fed colostrum (co) and particularly in pigs-fed formula (fo) when compared with pigs fed (PN) alone (iv). The only exception was TLR2. Methylation levels at the $H 19$ locus were about $50 \%$ in all cases reflecting its status as imprinted gene. Data are presented as median \pm interquartile ranges, minimum and maximum. All experiments were performed in technical triplicates.TPN: Total parenteral nutrition, PN: parenteral nutrition. 

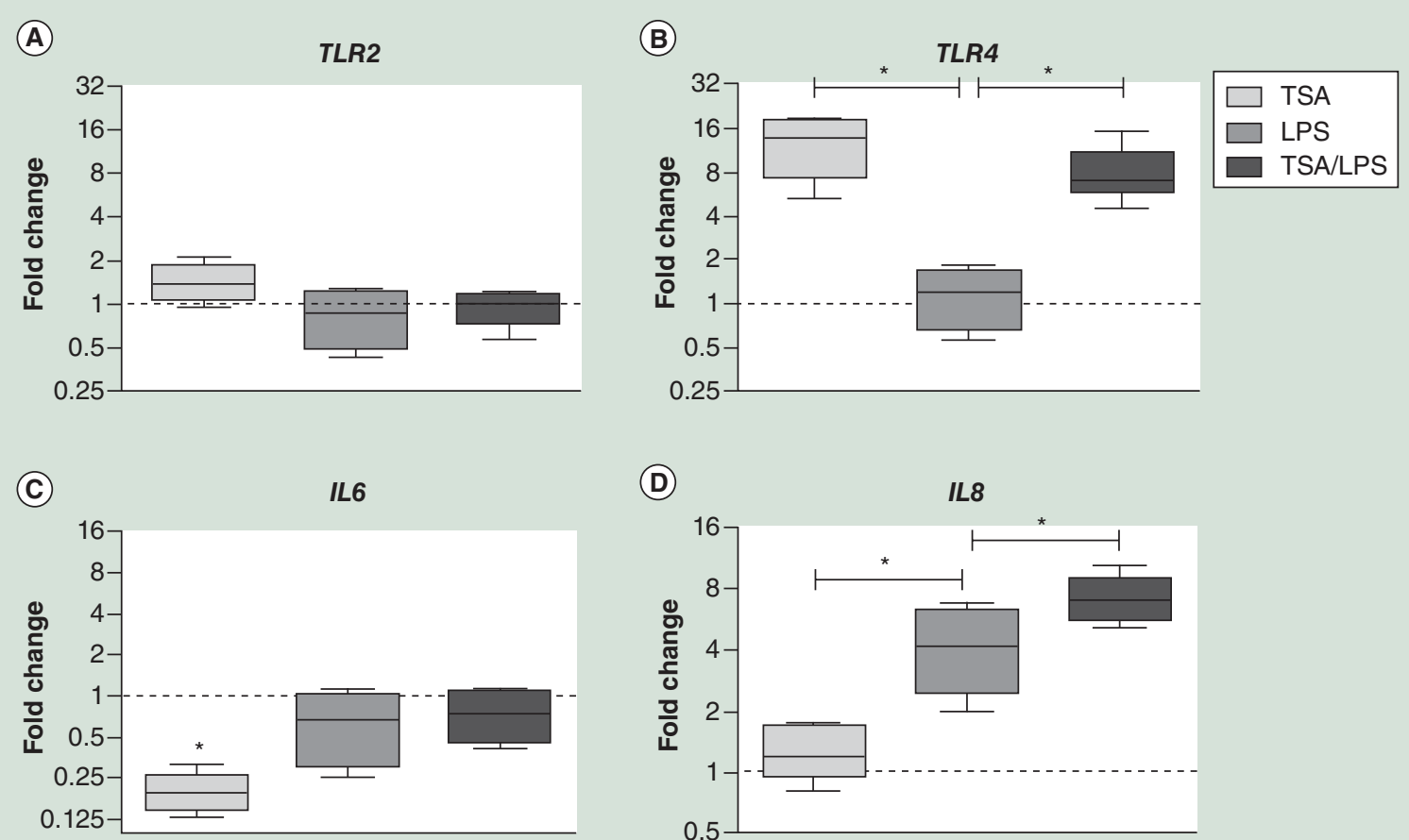

(D)

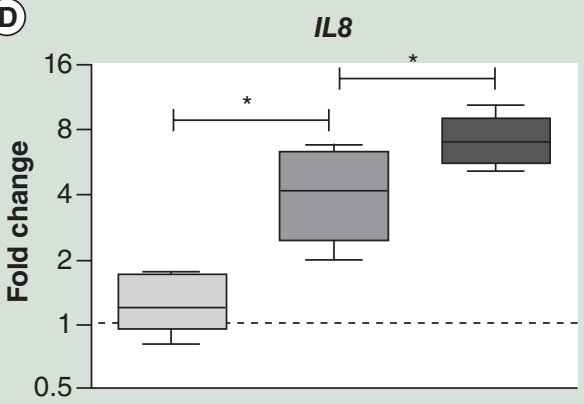

Figure 6. Inflammatory response upon lipopolysaccharides stimulation in CaCo-2 cells with or without treatment with trichostatin A. Upon histone deacetylase inhibition using TSA in CaCo-2 cells, TLR4 mRNA levels increased 12.7-fold whereas TLR2 and IL8 mRNA levels remained unchanged. Stimulation with LPS in naive CaCo-2 cells induced an increase in IL8 mRNA levels. This effect was significantly more pronounced in TSA pretreated CaCo2 cells. Data are presented as the median \pm interquartile range, minimum and maximum. All experiments were performed in technical triplicates. Asterisks indicate $p<0.05$

LPS: lipopolysaccharides; TSA: Trichostatin A.

reviewed the manuscript. A Jenke, P Sangild and J Postberg took part in the main study design, data analyses and supervision of the study. $L$ Krych was responsible for the microbiota analyses. A Jenke had primary responsibility for final content. All authors read and approved the final manuscript.

\section{Open access}

This work is licensed under the Creative Commons AttributionNonCommercial 3.0 Unported License. To view a copy of this license, visit http://creativecommons.org/licenses/by-nc-nd/3.0/

\section{Acknowledgements}

The authors would like to thank C Förster for her excellent technical support.

\section{Financial \& competing interests disclosure}

This study was funded by the HELIOS Research Center and the NEOMUNE research program from the Danish Research Councils. University of Copenhagen has filed a patent concerning the use of bovine colostrum for groups of pediatric patients. The authors have no other relevant affiliations or financial involvement with any organization or entity with a financial inter-

\section{Executive summary}

- Enteral nutrition induces subclinical intestinal epithelial inflammation

- Histopathological inflammation is more pronounced using formula feeding.

- Lactase activity was markedly reduced in the pigs supplemented with formula.

- Nutrition induces diet-specific changes in gene expression and chromatin signatures of genes related to inflammation and innate immunity

- Upregulation of several inflammatory and pattern recognition receptor genes correlates with histopathology and is more pronounced in formula feeding.

- Only colostrum induces expression of IL10 possibly lowering necrotizing enterocolitis susceptibility.

- Changes in gene expression translate to diet-specific changes in chromatin conformation.

- Chromatin decompaction increases Toll-like receptor 4 expression and lipopolysaccharides responsiveness in vitro. 
est in or financial conflict with the subject matter or materials discussed in the manuscript apart from those disclosed.

No writing assistance was utilized in the production of this manuscript.

\section{Ethical conduct of research}

This study was conducted with approval of the Witten/Herdecke University Ethics board. All animal procedures were ap-

\section{References}

Papers of special note have been highlighted as:

- of interest; $\bullet \bullet$ of considerable interest.

1 Neu J, Walker WA. Necrotizing enterocolitis. N. Engl. J. Med. 364(3), 255-264 (2011).

2 Zoppelli L, Guttel C, Bittrich HJ, Andree C, Wirth S, Jenke A. Fecal calprotectin concentrations in premature infants have a lower limit and show postnatal and gestational age dependence. Neonatology 102(1), 68-74 (2012).

3 Dabritz J, Jenke A, Wirth S, Foell D. Fecal phagocytespecific S100A12 for diagnosing necrotizing enterocolitis. J. Pediatr. 161(6), 1059-1064 (2012).

4 Jenke AC, Zilbauer M, Postberg J, Wirth S. Human beta-defensin 2 expression in ELBW infants with severe necrotizing enterocolitis. Pediatr. Res. 72(5), 513-520 (2012).

5 Wright NJ, Thyoka M, Kiely EM et al. The outcome of critically ill neonates undergoing laparotomy for necrotising enterocolitis in the neonatal intensive care unit: a 10-year review. J. Pediatr. Surg. 49(8), 1210-1214 (2014).

6 Lin PW, Stoll BJ. Necrotising enterocolitis. Lancet 368(9543), 1271-1283 (2006).

7 Henry MC, Moss RL. Necrotizing enterocolitis. Annu. Rev. Med. 60, 111-124 (2009).

-. This review provides a comprehensive summary on necrotizing enterocolitis (NEC) from the clinical perspective.

8 Claud EC, Walker WA. Hypothesis: inappropriate colonization of the premature intestine can cause neonatal necrotizing enterocolitis. FASEB J. 15(8), 1398-1403 (2001).

9 Nanthakumar NN, Fusunyan RD, Sanderson I, Walker WA. Inflammation in the developing human intestine: a possible pathophysiologic contribution to necrotizing enterocolitis. Proc. Natl Acad. Sci. USA 97(11), 6043-6048 (2000).

10 Nanthakumar NN, Young C, Ko JS et al. Glucocorticoid responsiveness in developing human intestine: possible role in prevention of necrotizing enterocolitis. Am. J. Physiol. Gastrointest. Liver Physiol. 288(1), G85-G92 (2005).

11 Lotz M, Gutle D, Walther S, Menard S, Bogdan C, Hornef MW. Postnatal acquisition of endotoxin tolerance in intestinal epithelial cells. J. Exp. Med. 203(4), 973-984 (2006).

12 Leaphart CL, Cavallo J, Gribar SC et al. A critical role for TLR 4 in the pathogenesis of necrotizing enterocolitis by proved by the National Council on Animal Experimentation in Denmark (protocol number 2012-15-293400193). The authors state that they have obtained appropriate institutional review board approval or have followed the principles outlined in the Declaration of Helsinki for all human or animal experimental investigations. In addition, for investigations involving human subjects, informed consent has been obtained from the participants involved.

modulating intestinal injury and repair. J. Immunol. 179(7), 4808-4820 (2007).

13 Jilling T, Simon D, Lu J et al. The roles of bacteria and TLR4 in rat and murine models of necrotizing enterocolitis. J. Immunol. 177(5), 3273-3282 (2006).

14 Sodhi CP, Shi XH, Richardson WM et al. Toll-like receptor- 4 inhibits enterocyte proliferation via impaired beta-catenin signaling in necrotizing enterocolitis. Gastroenterology 138(1), 185-196 (2010).

15 Yazji I, Sodhi CP, Lee EK et al. Endothelial TLR4 activation impairs intestinal microcirculatory perfusion in necrotizing enterocolitis via eNOS-NO-nitrite signaling. Proc. Natl Acad. Sci. USA 110(23), 9451-9456 (2013).

-• This excellent study provides the first data how breast milk might possibly alleviate NEC in the rodent model.

16 Chan KY, Leung KT, Tam YH et al. Genome-wide expression profiles of necrotizing enterocolitis versus spontaneous intestinal perforation in human intestinal tissues: dysregulation of functional pathways. Ann. Surg. 260(6), 1128-1137 (2014).

- The authors provide the first genome-wide association study (GWAS) data on human intestinal tissue comparing NEC with spontaneous intestinal perforation.

17 Bering SB, Bai S, Zhang K, Sangild PT. Prematurity does not markedly affect intestinal sensitivity to endotoxins and feeding in pigs. Br. J. Nutr. 108(4), 672-681 (2012).

18 Goldman AS. Modulation of the gastrointestinal tract of infants by human milk. Interfaces and interactions. An evolutionary perspective. J. Nutr. 130(2S Suppl.), S426-S431 (2000).

19 Sangild PT, Siggers RH, Schmidt M et al. Diet- and colonization-dependent intestinal dysfunction predisposes to necrotizing enterocolitis in preterm pigs. Gastroenterology 130(6), 1776-1792 (2006).

-• The study describes the currently best animal model for NEC.

20 Bjornvad CR, Schmidt M, Petersen YM et al. Preterm birth makes the immature intestine sensitive to feeding-induced intestinal atrophy. Am. J. Physiol. Regul. Integr. Comp. Physiol. 289(4), R1212-R1222 (2005).

21 Sangild PT, Thymann T, Schmidt M, Stoll B, Burrin DG, Buddington RK. Invited review: the preterm pig as a model in pediatric gastroenterology. J. Animal Sci. 91(10), 4713-4729 (2013).

22 Bjornvad CR, Thymann T, Deutz NE et al. Enteral feeding induces diet-dependent mucosal dysfunction, bacterial 
proliferation, and necrotizing enterocolitis in preterm pigs on parenteral nutrition. Am. J. Physiol. Gastrointest. Liver Physiol. 295(5), G1092-G1103 (2008).

https://clinicaltrials.gov/ct2/show/NCT02054091

24

Pyndt Jorgensen B, Hansen JT, Krych L et al. A possible link between food and mood: dietary impact on gut microbiota and behavior in BALB/c mice. PloS ONE 9(8), e103398 (2014).

25 Livak KJ, Schmittgen TD. Analysis of relative gene expression data using real-time quantitative PCR and the 2(-Delta Delta C(T)) Method. Methods 25(4), 402-408 (2001).

26 Drewell RA, Goddard CJ, Thomas JO, Surani MA. Methylation-dependent silencing at the H19 imprinting control region by MeCP2. Nucleic Acids Res. 30 (5), 1139-1144 (2002).

27 Grontved L, Bandle R, John S et al. Rapid genome-scale mapping of chromatin accessibility in tissue. Epigenetics Chromatin 5(1), 10 (2012).

- This study describes the tissue accessible chromatin methodology, a robust method to identify accessible chromatin, applicable in a broad range of clinically relevant samples.

28 Thurman RE, Rynes E, Humbert R et al. The accessible chromatin landscape of the human genome. Nature 489(7414), 75-82 (2012)

29 Lai Y, Li D, Li C et al. The antimicrobial protein REG3A regulates keratinocyte proliferation and differentiation after skin injury. Immunity 37(1), 74-84 (2012).

30 Grasset E, Pinto M, Dussaulx E, Zweibaum A, Desjeux JF Epithelial properties of human colonic carcinoma cell line Caco-2: electrical parameters. Am. J. Physiol. 247(3 Pt 1), C260-C267 (1984).

31 Sambuy Y, De A, I, Ranaldi G, Scarino ML, Stammati A, Zucco F. The Caco-2 cell line as a model of the intestinal barrier: influence of cell and culture-related factors on Caco2 cell functional characteristics. Cell Biol. Toxicol. 21(1), $1-26$ (2005).

32 Lucas A, Cole TJ. Breast milk and neonatal necrotising enterocolitis. Lancet 336(8730), 1519-1523 (1990).

33 Ramani M, Ambalavanan N. Feeding practices and necrotizing enterocolitis. Clin. Perinatol. 40(1), 1-10 (2013).

34 Cilieborg MS, Boye M, Thymann T, Jensen BB, Sangild PT. Diet-dependent effects of minimal enteral nutrition on intestinal function and necrotizing enterocolitis in preterm pigs. JPEN J. Parenter. Enteral Nutr. 35(1), 32-42 (2011).

35 Thymann T, Moller HK, Stoll B et al. Carbohydrate maldigestion induces necrotizing enterocolitis in preterm pigs. Am. J. Physiol. Gastrointest. Liver Physiol. 297(6), G1115-G1125 (2009).

36 Jeltsch A, Jurkowska RZ. New concepts in DNA methylation. Trends Biochem. Sci. 39(7), 310-318 (2014).

37 Hackam DJ, Afrazi A, Good M, Sodhi CP. Innate immune signaling in the pathogenesis of necrotizing enterocolitis. Clin. Dev. Immunol. 2013475415 (2013)
- This review summarizes the current evidence for a Toll-like receptor 4-driven exaggerated immune response as key factor for the development of NEC.

38 Gribar SC, Sodhi CP, Richardson WM et al. Reciprocal expression and signaling of TLR4 and TLR9 in the pathogenesis and treatment of necrotizing enterocolitis. J. Immunol. 182(1), 636-646 (2009).

39 Heneghan AF, Pierre JF, Tandee K et al. Parenteral nutrition decreases paneth cell function and intestinal bactericidal activity while increasing susceptibility to bacterial enteroinvasion. JPEN J. Parenter. Enteral Nutr. 38(7), 817-824 (2013).

40 Mcelroy SJ, Underwood MA, Sherman MP. Paneth cells and necrotizing enterocolitis: a novel hypothesis for disease pathogenesis. Neonatology 103(1), 10-20 (2013).

41 Morgan J, Bombell S, Mcguire W. Early trophic feeding versus enteral fasting for very preterm or very low birth weight infants. Cochrane Database Syst. Rev. 3, CD000504 (2013).

42 Bartuzi P, Hofker MH, Van De Sluis B. Tuning NF-kappaB activity: a touch of COMMD proteins. Biochim. Biophys. Acta 1832 (12), 2315-2321 (2013).

43 Cilieborg MS, Schmidt M, Skovgaard K et al. Fetal lipopolysaccharide exposure modulates diet-dependent gut maturation and sensitivity to necrotising enterocolitis in preterm pigs. Br. J. Nutr. 106(6), 852-861 (2011).

44 Lucas A, Cole TJ. Breast milk and necrotising enterocolitis. Lancet 336(8730), 1519-1523 (1990).

45 Siggers J, Sangild PT, Jensen TK et al. Transition from parenteral to enteral nutrition induces immediate dietdependent gut histological and immunological responses in preterm neonates. Am. J. Physiol. Gastrointest. Liver Physiol. 301(3), G435-G445 (2011).

46 Schaedler RW, Dubos R, Costello R. The development of the bacterial flora in the gastrointestinal tract of mice. J. Exp. Med. 122, 59-66 (1965).

47 Cilieborg MS, Boye M, Sangild PT. Bacterial colonization and gut development in preterm neonates. Early Hum. Dev 88(Suppl. 1), S41-S49 (2012).

48 Berg RD. The indigenous gastrointestinal microflora. Trends Microbiol. 4(11), 430-435 (1996).

49 Codd R, Braich N, Liu J, Soe CZ, Pakchung AA. Zn(II)dependent histone deacetylase inhibitors: suberoylanilide hydroxamic acid and trichostatin A. Int. J. Biochem. Cell Biol. 41(4), 736-739 (2009)

50 Park BS, Lee JO. Recognition of lipopolysaccharide pattern by TLR4 complexes. Exp. Mol. Med. 45, e66 (2013).

51 Calatayud M, Gimeno-Alcaniz JV, Velez D, Devesa V. Trivalent arsenic species induce changes in expression and levels of proinflammatory cytokines in intestinal epithelial cells. Toxicol. Lett. 224(1), 40-46 (2014).

52 Larsson L, Thorbert-Mros S, Rymo L, Berglundh T. Influence of epigenetic modifications of the interleukin-10 promoter on IL10 gene expression. Eur. J. Oral Sci. 120(1), 14-20 (2012).

53 Poplutz MK, Wessels I, Rink L, Uciechowski P. Regulation of the interleukin- 6 gene expression during monocytic differentiation of HL-60 cells by chromatin remodeling and methylation. Immunobiology 219(8), 619-626 (2014). 